\title{
Orchestrated SDN-based VDC Provisioning over Multi- Technology Optical Data Centre Networks
}

\author{
Salvatore Spadaro, Senior Member, IEEE, Albert Pagès, Fernando Agraz, Rafael Montero, Jordi Perelló \\ Advanced Broadband Communications Centre (CCABA), Universitat Politècnica de Catalunya (UPC) \\ Jordi Girona 1-3, 08034 Barcelona, Spain \\ Email: spadaro@tsc.upc.edu
}

\begin{abstract}
In this paper, we describe some scenarios and technologies that have been proposed to cope with the requirements of current and next generation data centre infrastructure. In particular, we discuss the extensions that have been implemented at both orchestration and control levels to efficiently manage the data centres resources. We put the focus on the integration between the Orchestrator and the SDN Controller by describing the communication interfaces and their interaction to provision optimized Virtual Data Centres (VDC) instances over novel data centre infrastructure, with special mention to the different solutions adopted to manage multiple optical technologies at the data plane.
\end{abstract}

Keywords: SDN, Orchestration, DCN.

\section{INTRODUCTION}

Current data centre (DC) infrastructure providers need to cope with the constantly growing number of customers (tenants) and services that are executed in their premises. Furthermore, isolation between tenants and committed Quality of Service (QoS) have to be guaranteed. In this context, the Virtual Data Centre (VDC) paradigm, where a DC provider rents parts of its physical infrastructure to different customers who, in turn, develop their own business models, arises as an appropriate solution [1]. Since the VDC provisioning requires the selection of the most suitable IT resources (Virtual Machines (VMs)) placement onto physical servers and the configuration of the network to interconnect them, the automation of the process becomes paramount to optimize the usage of the DC physical resources as well as to deal with its complexity and slowness.

In light of this, the COSIGN project [2] was funded by the EU to study and implement a novel DC infrastructure able to dynamically provide DC virtualization services over a new generation optical data plane. The project worked on the three architectural planes, namely data, control and orchestration, and integrated them by means of standards-based interfaces. In the data plane, two different scenarios were defined: medium term and long term. While the former used hybrid electrical/optical transport technologies providing low latency and high radix, the latter was based on pure optical transport, which is foreseen as the most suitable solution to also provide ultra-high bandwidth and low power consumption. This paper describes the integrated control and orchestration of both data plane architectures and paves the way to the provisioning of VDC instances over a heterogeneous multi-technology optical DC network infrastructure.

\section{CONTROL AND ORCHESTRATION PLANES}

The SDN Controller and the Orchestrator are responsible for the network and IT resources configuration, respectively. Although they have different and well-defined responsibilities, both entities have to cooperate to provide virtualization and application services in an effective way and guaranteeing an efficient usage of the DC resources, as well.

The SDN Controller (Figure 1, bottom-left) is based on OpenDaylight Lithium [3] and is responsible for configuring the DCN network. To this end, the Controller implements a set of modules to collect data plane information, to compute routes over the data plane and to configure it to provide connectivity between servers. More specifically, the OpenFlow (OF) Plugin and OF Java modules implement the OF-based SouthBound (SB) interface, which enables the communication between the Controller and the data plane devices. This interface is used by the Controller to collect topological and inventory information from the network. The upper layer modules of the Controller use this information for their purposes. Hence, the Path Computation Manager (PCM) and the Optical Provisioning Manager (OPM) use this information to compute routes and configure the optical data plane, respectively. The Optical Resource Virtualization Manager (ORVM) uses it to map the virtual network infrastructure associated to a service over the physical data plane. Finally, the Virtual Tenant Network (VTN) uses the topological information to configure the level 2 connectivity of the virtual network.

Besides, the Controller communicates with the Orchestrator, by means of the NorthBound (NB) interface, for a synchronized network plus IT resources configuration. In particular, the VTN is responsible for receiving the network configuration request associated to a service that is being configured through the Orchestrator. The VTN then contacts the ORVM, which is responsible to manage the mapping of the requested virtual network over the physical data plane. To do this, the ORVM contacts the OPM, which configures the optical data plane 
through the above-mentioned OF Plugin. The route has been previously sent to the OPM by the PCM. Further detail on the whole provisioning process is given in next section.

The Orchestrator (Figure 1, top-left) is based on OpenStack [4], which provides an open platform for management of cloud infrastructures. Through the collaborative efforts of multiple modules, it allows for the creation of virtual infrastructures (named stacks) satisfying the requirements of external users (tenants) in regards of both computing and networking resources. For this, the Nova service takes care of the instantiation of the computing resources (e.g. VMs) and the Neutron service configures the IP virtual network, while the Heat service coordinates both of them thanks to a template-driven workflow. To cope with the joint IT and network provisioning, and tailor the process for the particularities of the physical technology at the DC network (DCN), we extended OpenStack with an Algorithms module to compute the optimal VDC mapping. The Algorithms module contacts the Controller to collect topological information and the OpenStack Nova service to get information about the IT resources to realize the VDC layout computation. With such information, it determines the VM mapping onto servers and the virtual network mapping onto connections at the optical DCN. The decided mapping is enforced by constructing a Heat template containing the desired mapping of the VMs while contacting with the Controller for the route specification and configuration.

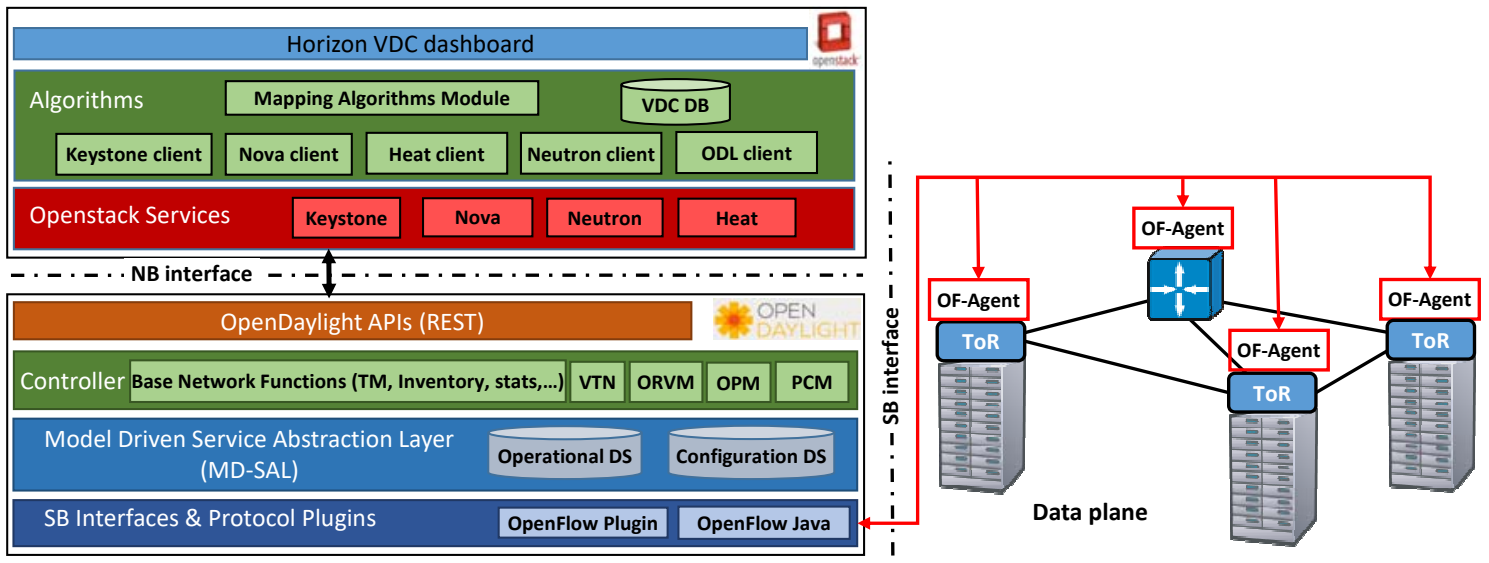

Figure 1. COSIGN project Control and Orchestration planes architecture.

Furthermore, the Orchestrator and the SDN Controller have been designed to enable the configurability of the diverse data plane technologies associated to the different (medium and long term) transport solutions. In the medium term scenario, the servers hosting the compute nodes of a rack are connected to a high radix and low latency electronic Top of the Rack (ToR) switch Error! Reference source not found.. The ToRs are then optically interconnected by means of a Large Port count Fibre Switch (LPFS). On the other hand, in the long term data plane, the physical servers are equipped with an optical Network Interface Card (NIC) capable of sending traffic using both Time Division Multiplexing (TDM) and Optical Circuit Switching (OCS) optical technologies. Hence, the TDM output of the NIC is connected to a TDM fast switch, and the OCS one is connected to a LPFS [6]. The following section provides further detail on the particularities of the provisioning over each data plane infrastructure, while demonstrating the integration of the SDN Controller and the Orchestrator.

\section{VDCS ALLOCATION}

The VDC allocation process is detailed in Figure 2. Such process starts from the Orchestrator, which is used by the DC operator to fulfil VDC requests from tenants. In particular, the operator accesses to the dashboard that provides a graphical interface to configure the features of the VDC (step 1). Once the VDC has been designed and validated, the dashboard contacts the Algorithms module (Figure 2, step 2), which uses topological information previously collected from the Topology Manager (TM) of the Controller to compute an optimized layout of the IT resources and the physical paths associated to the virtual links that inter-connect them. The Algorithms module then triggers the creation of the stack (i.e. the collection of virtual resources, such as the IP network and the VMs) by contacting the Heat module of OpenStack, passing down a template containing the details of the stack (Figure 2, step 3). Figure 3 depicts a sample of traffic through the interface developed to communicate the Algorithms module and the OpenStack core. The template sent from the Algorithms contains both the VM placement and the IP network configuration of the VDC. With this, the Nova module of the Orchestrator employs the VM related information to deploy the IT infrastructure (Figure 2, step 5), and the Neutron module sends the network information to the Controller (step 4). The Controller receives the network creation request in the VTN module, which will create the IP network associated to the VDC. At this point, when the IP network has been created and the VMs have been spawned and booted up, the VDC is accessible to the 
users. Nonetheless, the physical paths associated to the virtual links that interconnect the VMs have not been configured yet. Following the SDN paradigm, these paths are configured when the communication between VMs is established, that is, when traffic exchanges (e.g. IP) between VMs are required. Once the first traffic packet reaches the OVS L2 virtual switch that connects the compute node to the data plane (i.e. the TOR), this OVS sends the packet to the Controller where it is processed by the VTN (steps 6 and 7 in Figure 2). The VTN contacts the ORVM, which triggers the configuration of the physical paths associated to the virtual links that connect the VMs. The ORVM requests the path provisioning to the OPM, which in turn requests the path to the PCM. In our orchestrator-based approach, instead of computing the path, the PCM requests it to the Algorithms module of the Orchestrator (Figure 2, step 10). Once it receives the route, the PCM forwards it to the OPM. Finally, the OPM configures the flows in the physical network devices involved in the path by means of the OF plugin module (step 11b) and the VTN configures the L2 flows in the OVS instances of the compute nodes (step 11a). Figure 4 illustrates the OF-based SB interface communication between the Controller and the data plane. Once the data plane is configured, traffic exchanges can be realized through the provisioned VDC slice.

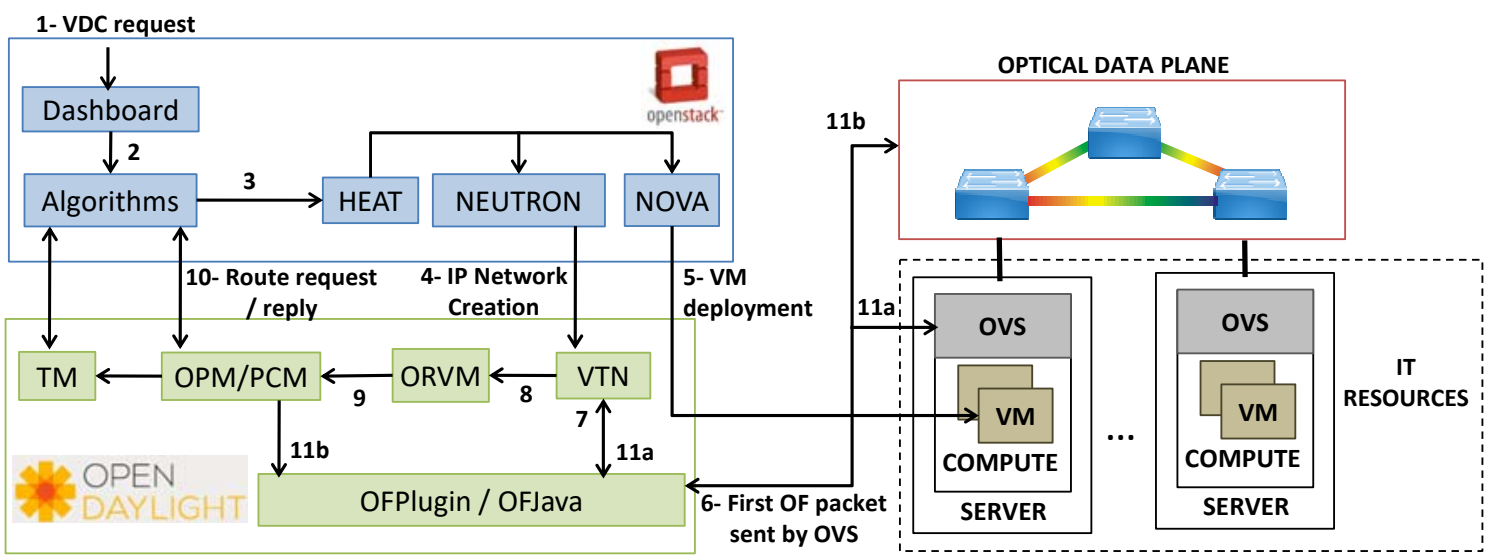

Figure 2. General VDC allocation workflow.

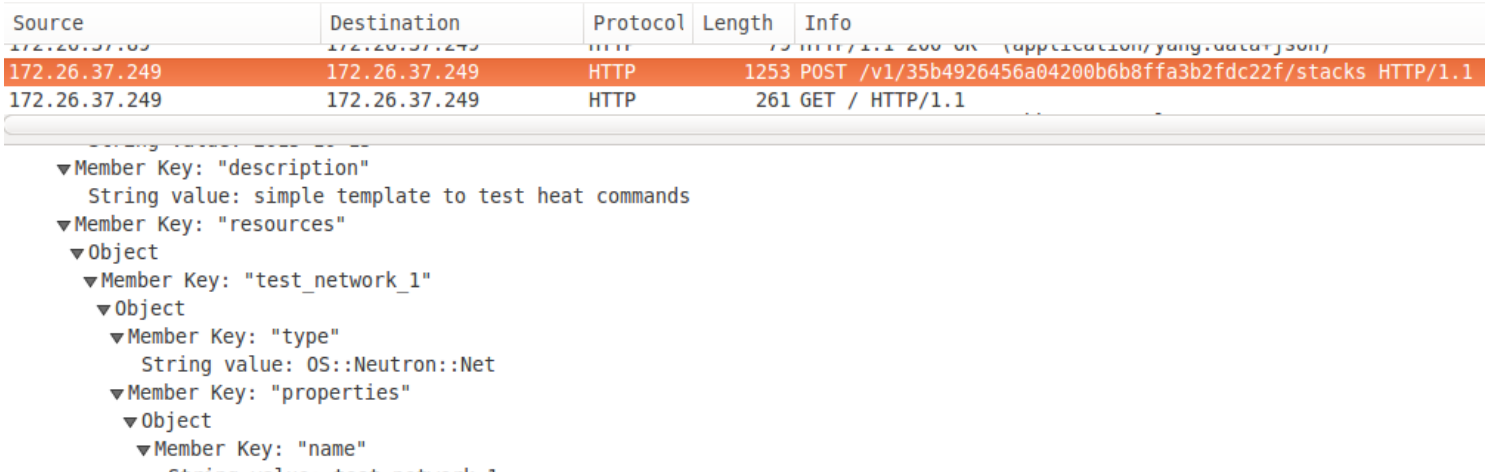

Figure 3. REST interface between the Algorithms module and the Heat module of OpenStack.

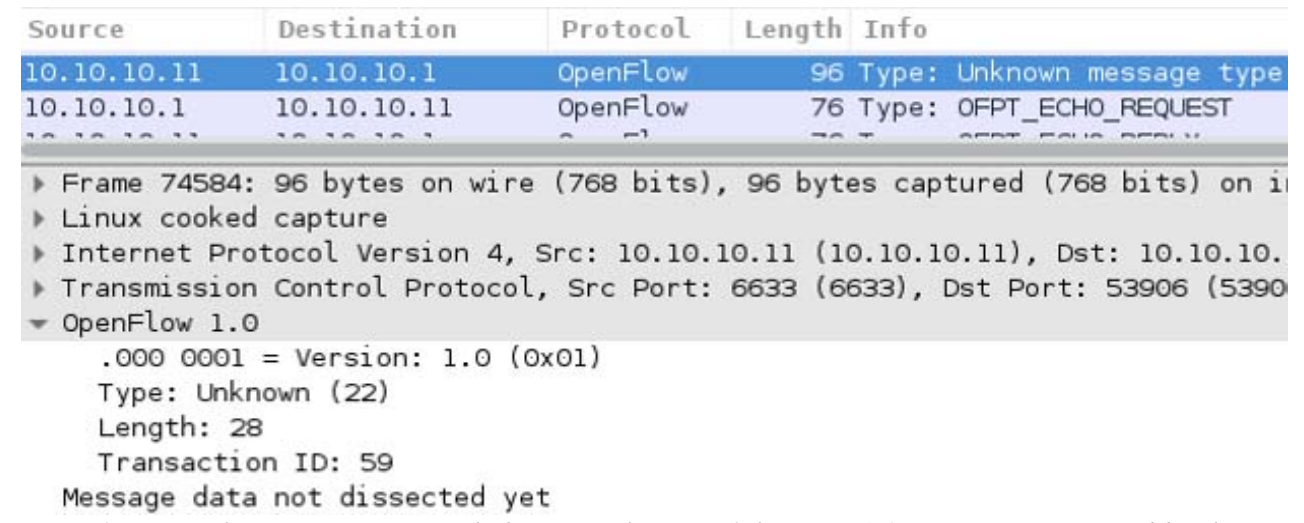

Figure 4. OF CFlow message extended in compliance of the OF v1.0 OCS extensions addendum [7]. 


\section{VDC ALLOCATION OVER MULTI-TECHNOLOGY OPTICAL DCNS}

In the previous sections, we have detailed the VDC provisioning over a generic architecture of the EU COSIGN project. Nonetheless, the current design and implementation of both the SDN Controller and the Orchestrator would be capable to manage a heterogeneous multi-technology DCN infrastructure. In particular, at the Controller level, the OF-based modules are able to communicate to the physical devices of both the medium and the long term scenarios. In the same way, the rest of the modules of the Controller have been extended to manage network connections using either of the network elements. Finally, the Algorithms module of the Orchestrator is able to compute VDC instances using heterogeneous network infrastructures.

Particularly, TDM or OCS-based connectivity can be achieved thanks to the developed extensions to support the configuration of the hybrid technologies. First, the OF Plugin and OF Java modules of the Controller were extended to configure the novel network elements (i.e. the optical NIC). Similarly, the TM, the OPM and the PCM were updated to maintain, configure and compute routes over the long term data plane, respectively. Finally, the ORVM was modified to manage the virtualization of the new network elements. At the orchestration level, the Algorithms module was extended to compute optimized joint IT plus network VDC computation over the presence of TDM/OCS technologies. In addition, the communication between the Algorithms module and the OPM was updated to cope with the specific features of TDM routes. Figure 5 illustrates the different characteristics of the routes computed over OCS (left) and TDM (right) technologies. In conclusion, the developed software paves the way for the management and configuration of multi-technology DCN scenarios, opening the possibility to choose the most suitable one in support of heterogeneous VDC instances.

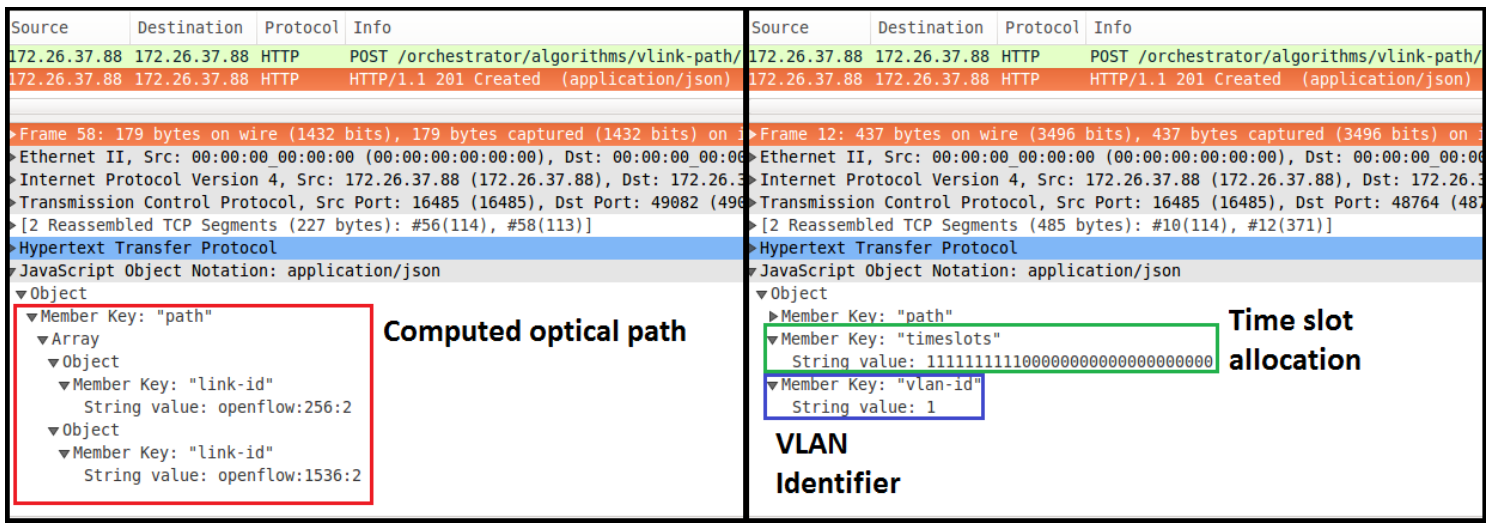

Figure 5. RESTful route reply from the Orchestrator to the SDN Controller for OCS (left) and TDM (right) technologies.

\section{CONCLUSIONS}

In this paper we presented the experimental integration of the orchestration and control layers to provide optimized VDC instances in future data centres. We highlighted how the developed SDN Controller and the Orchestrator are able to manage a heterogeneous multi-technology optical data plane, allowing for optimized selection of resources as a function of the VDC characteristics.

\section{ACKNOWLEDGEMENTS}

This work has been supported by the EU FP7 COSIGN project (FP7-619572) and the Spanish Government through project SUNSET (TEC2014-59583-C2-1-R) with FEDER contribution.

\section{REFERENCES}

[1] A.Pagès, et al.: Experimental assessment of VDC provisioning in SDN/OpenStack-based DC infrastructures with optical DCN, in Proc. ECOC 2016, Dusseldorf, Germany, September 2016.

[2] EU FP7 COSIGN project, http://www.fp7-cosign.eu/

[3] OpenDaylight, https://www.opendaylight.org/

[4] OpenStack, https://www.openstack.org/

[5] Oded Raz, et al.: Optical Solutions for the Challenges of Mega-Size Data Center Networks, in Proc. OFC 2016, Anaheim, USA, March 2016.

[6] C. Jackson, et al.: Demonstration of the benefits of SDN technology for all-optical data centrevirtualisation, in Proc. OFC 2017, Los Angeles, USA, March 2017.

[7] Open Networking Foundation, Extensions to the OpenFlow Protocol in support of Circuit Switching, Addendum to OpenFlow Protocol Specification v1.0 - Circuit Switch Addendum v.0.3, June 2010 